\title{
Programa de acompanhamento de egressos e sua relação com os cursos superiores de Gestão Ambiental: análise do cenário da Região Sudeste do Brasil em 2016
}

\section{Alumni folow up program and its relationship at Environmental Management higher courses: an analysis of Southeastern Region of Brazil scenario in 2016}

Programa de seguimento de egresados y su relación con los cursos superiores de Gestión Ambiental: análisis del escenario de la Región Sudeste de Brasil en 2016

Valéria Garcez de Oliveira ${ }^{1}$ Luís Cláudio Meirelles de Medeiros ${ }^{2}$ Julianne Alvim Milward-de-Azevedo ${ }^{3}$

${ }^{1}$ Mestre em Tecnologia Ambiental da Universidade Federal Fluminense.

Técnica Ambiental pelo Instituto Federal do Rio de Janeiro. Gestora Ambiental pela Universidade Federal Rural do Rio de Janeiro. E-mail: valeria_garcez@outlook.com, Orcid: http://orcid.org/0000-0002-0867-809X

${ }^{2}$ Doutor pelo Programa de Pós-Graduação em Meio Ambiente da Universidade do Estado do Rio de Janeiro (UERJ). Mestre em Administração Pública pela

Escola Brasileira de Administração Pública e de Empresas (EBAPE/FGV). Graduado em Economia e Administração pela PUC/RJ. Professor Assistente do Departamento de Ciências do Meio Ambiente (DCMA/UFRRJ). E-mail: claudiommedeiros@hotmail.com, Orcid: http://orcid.org/0000-0002-8041-585X

${ }^{3}$ Doutora em Economia pela Universidade Federal do Rio de Janeiro (UFRJ). Mestre em Administração Pública pela Fundação Getúlio Vargas. Bacharel em Administração de Empresas pela Universidade Santa Úrsula, e em Arquitetura e Urbanismo pela Universidade Federal Fluminense. Professora

Associada da Universidade Federal Rural do Rio de Janeiro (UFRRJ), Departamento de Ciências do Meio Ambiente (DCMA). Coordenadora do Núcleo de Estudos sobre Trabalho, Políticas e Desenvolvimento (NETPD) e do Núcleo de Estudos em Gestão de Unidades de Conservação (NEGUC) na UFRRJ. E-mail: juliannemilward.UFRRJ@gmail.com, Orcid: http://orcid.org/0000-0001-9066-0843 
Resumo: A necessidade de um profissional na área ambiental caracterizado como multidisciplinar criou demandas para a criação do Gestor Ambiental; a partir disto, o presente estudo tem como objetivo apresentar o levantamento quantitativo dos cursos superiores de Gestão Ambiental presentes na região Sudeste brasileira, suas modalidades de ensino e categorias bem como a existência ou não do acompanhamento de seus egressos. O Programa de Acompanhamento de Egressos (PAE) é recomendado pelo Ministério da Educação. A região Sudeste, em 2016, possuía diversos cursos superiores em Gestão Ambiental, entretanto, a maioria desses cursos encontrava-se nos estados de São Paulo, Minas Gerais e Rio de Janeiro, respectivamente. A maior parte dos cursos era de instituições privadas de ensino e possuía a modalidade de ensino à distância, os cursos de bacharelado apresentaram-se em minoria. Em relação ao PAE de todos esses cursos, nenhuma instituição apresentou um acompanhamento de egressos específico para graduados em Gestão Ambiental.

Palavras-chave: programa de monitoramento de egressos; Ensino Superior; Gestão Ambiental; egressos.

Abstract: A professional's need in the environmental area characterized as multidisciplinar created demands for the creation of the Environmental Manager, starting from this, the present study has as objective presents the quantitative rising of the universities of Administration Environmental presents in the Brazilian Southeast area, their teaching modalities and categories as well as the existence or not of the attendance of their exits. The Alumni Follow Up Program (PAE) is recommended by Brazilian Education Ministry. The Southeast region in 2016 had several higher education courses in Environmental Management, however, most of these courses were in the states of São Paulo, Minas Gerais and Rio de Janeiro, respectively. Most courses came from private educational institutions and had the type for distance education; the bachelor's presented themselves in the minority. Regarding the PAE of all these courses, none institution presented specific alumni attendance for graduate in Environmental Administration.

Keywords: alumni follow up program; Higher Education; Environmental Management; graduates.

Resumen: La necesidad de un profesional en el área ambiental caracterizado como multidisciplinario creó demandas para la creación del Gestor Ambiental; a partir de esto, el presente estudio tiene como objetivo presentar el levantamiento cuantitativo de los cursos superiores de Gestión Ambiental presentes en la región Sudeste brasileña, sus modalidades de enseñanza y categorías así como la existencia o no del acompañamiento de sus egresados. El Programa de Seguimiento de Egresos (PAE) es recomendado por el Ministerio de Educación. La región Sudeste en 2016 poseía diversos cursos superiores en Gestión Ambiental, sin embargo, la mayoría de estos cursos se encontraban en los estados de São Paulo, Minas Gerais y Río de Janeiro, respectivamente. La mayor parte de los cursos eran de instituciones privadas de enseñanza y poseían la modalidad de enseñanza a distancia, los cursos de bachillerato se presentaron en minoría. En relación al PSE de todos estos cursos, ninguna institución presentó un acompañamiento de egresados específico para graduados en Gestión Ambiental.

Palabras clave: programa de monitoreo de egresados; Enseñanza Superior; Gestión Ambiental; graduados. 


\section{INTRODUÇÃO}

Desde os primórdios da humanidade, a antropização tem causado impactos irreversíveis ao meio ambiente, esses impactos acumulados ao longo de todos esses milhares de anos agravam gradativamente a situação do planeta Terra; ao utilizar, de forma demasiada, recursos naturais e lançar na atmosfera e superfície substâncias poluentes, a ação antrópica causou deficiência e esgotamento ao planeta Terra.

A crise ambiental ocorre em virtude da utilização irracional e ilimitada dos recursos naturais, que parte do pressuposto de que esses recursos são infinitos. A sociedade contemporânea possui hábitos que impulsionam o consumo demasiado dos recursos que a Terra nos oferece. Dessa forma, o ritmo de consumo e utilização torna-se maior do que a taxa de recomposição desses recursos.

A geração de resíduos, a emissão de poluentes atmosféricos, a contaminação hídrica e do solo geram, portanto, um contexto caracterizado por uma crise geral que envolve consequências na economia, na política e na saúde pública.

Neste trabalho, apresentam-se algumas definições de meio ambiente. A Resolução do Conselho Nacional de Meio Ambiente (CONAMA) n. 306 de 2002 define meio ambiente como o conjunto de condições, leis, influência e interações de ordem física, química, biológica, social, cultural e urbanística, que permite, abriga e rege a vida em todas as suas formas. A definição de meio ambiente para Miller Junior (2014, p. 3) constitui-se de tudo o que afeta um organismo vivo (qualquer forma de vida única). Já Barbieri (2007, p. 5) define meio ambiente da seguinte forma:

Assim, por meio ambiente se entende o meio ambiente natural e o artificial, isto é, o ambiente físico e biológico originais e o que foi alterado, destruído e construído pelos humanos, como as áreas urbanas, industriais e rurais. Esses elementos condicionam a existência dos seres vivos, podendo-se dizer, portanto, que o meio ambiente, não é apenas o espaço onde os seres vivos existem ou podem existir, mas a própria condição de existência de vida na Terra.

A definição de ambiente apresentada por Sánchez (2013, p. 18) corrobora com a amplitude do termo desta forma: 
O conceito de "ambiente", no campo do planejamento e gestão ambiental é amplo, multifacetado e maleável. Amplo porque pode incluir tanto a natureza como a sociedade. Multifacetado porque pode ser apreendido sob diferentes perspectivas. Maleável porque, ao ser amplo e multifacetado, pode ser reduzido ou ampliado de acordo com necessidades do analista ou interesses dos envolvidos.

Considerando as definições apresentadas acima, é possível inferir que o meio ambiente não se resume apenas à flora e à fauna, mas também se incluem os fenômenos de ordem social e cultural. Dessa forma, o meio ambiente torna-se algo complexo e estruturado por diferentes ligações interdependentes que envolvem questões variadas, e os impactos causados ao meio natural refletem em questões econômicas, sociais, políticas e de saúde.

Sendo assim, a partir dessas características extremamente variadas de meio ambiente e da demanda emergente em criar soluções sustentáveis e formas diferentes de desenvolvimento, surge a necessidade de um profissional estruturado em bases multidisciplinares e capacitado para mediar e solucionar conflitos atuais de cunho ambiental abrangendo todos os seus diversificados aspectos: o gestor ambiental.

O presente estudo possui enfoque no levantamento quantitativo dos cursos superiores de Gestão Ambiental, em todas as suas modalidades, na região sudeste brasileira, em 2016. Além disso, também objetivou analisar a existência de acompanhamento de egressos por parte das instituições de ensino; esse acompanhamento caracteriza-se como recomendação do Ministério da Educação (MEC).

O acompanhamento do egresso é muito importante no que tange à avaliação do curso pelo mercado de trabalho, mostrando que o curso deve estar atento às necessidades desse mercado e deve, em tempo, formar um profissional capacitado para atender suas demandas.

Além disso, manter o contato com o graduado pode oferecer informações para a coordenação do curso que os graduandos não conseguem visualizar, o egresso pode apresentar deficiências e lacunas de assuntos que lhe foram cobrados no mercado e que a instituição não ofereceu como aprendizado. Lousada e Martins (2005, p.74) também destacam a importância do acompanhamento de egresso: 
É, pois, imprescindível saber o que os egressos pensam a respeito da formação recebida para se proceder a ajustes em todas as partes do sistema de ensino ofertado. Além disso, conhecer o que fazem como profissionais e cidadãos e suas adequações aos setores em que atuam, possibilita uma reflexão crítica sobre a formação e sua relação com as necessidades do mercado de trabalho.

O egresso pode, então, ser um intermediário da relação mercado-universidade e, dessa forma, estreitar relações e mostrar à Academia as falhas que precisam ser resolvidas para suprir totalmente a demanda do mercado de trabalho.

Assim, a relevância desse Programa de Acompanhamento de Egressos encontra-se na necessidade de autoavaliação da Instituição e dos processos de acesso à qualidade da formação oferecida. Nesse sentido, as informações obtidas oferecem subsídios aos Relatórios de Avaliação Institucional desenvolvidos. De igual importância, o Programa possibilita a realização da verificação continuada da adequação das matrizes curriculares às demandas sociais e econômicas.

\section{METODOLOGIA}

A pesquisa realizada caracteriza-se pelo seu caráter exploratório, analítico-descritivo. Quanto aos processos, foram utilizados, para a sua investigação, a pesquisa bibliográfica, documental, além de se constituir em um estudo de caso, em virtude de o tema abordado apresentar-se restrito aos programas de acompanhamento de egressos na Região Sudeste do Brasil, no ano de 2016.

Na pesquisa bibliográfica, foi realizado um levantamento das produções científicas referentes aos cursos superiores de Gestão Ambiental no Brasil e na região sudeste. A pesquisa documental foi dada a partir da investigação dos programas de acompanhamento de egressos presentes nos sítios eletrônicos das instituições de ensino do cur8uso em Gestão Ambiental, de todas as modalidades de ensino superior, na região sudeste brasileira, no período compreendido entre novembro de 2015 a abril de 2016.

Além disso, foram examinadas as regulamentações do Ministério da Educação quanto aos programas de acompanhamento de egressos. No Portal E-mec, foram analisados os registros e cadastros de todos os cursos 
superiores no Brasil, incluindo as diferentes modalidades dos cursos em Gestão Ambiental, da região sudeste, e coletados os dados relevantes para o desenvolvimento do estudo.

Os dados coletados do Portal E-mec foram tratados de forma qualitativa - por meio da codificação -, e de forma quantitativa - por meio do auxílio do programa Excel - planilhas eletrônicas -, com vista à geração dos quadros.

É importante destacar que esta pesquisa realizada no 1 ㅇ semestre de 2016, resultou no cenário da região sudeste para aquele momento. No ano de 2018, tem-se a expectativa do fechamento de um novo levantamento dos dados, para o desenvolvimento de uma análise temporal comparativa do cenário atual frente ao cenário de 2016.

\section{RESULTADOS}

\subsection{O contexto dos cursos superiores em Gestão Ambiental}

Barbieri (2007, p. 25) define a gestão ambiental como as ferramentas administrativas que possuam o objetivo de gerar resultados benéficos ao meio ambiente, evitando ou minimizando os impactos ambientais causados pelas ações antrópicas.

As instituições de ensino superior têm a função de realizar pesquisas e formar profissionais a fim de atender as demandas da sociedade; dessa forma, por conta da crise ambiental e da necessidade de conciliação entre desenvolvimento econômico e preservação ambiental, surge uma nova categoria profissional de gestores ambientais (LEANDRO, 2013, p. 19).

Como exemplo, pode-se citar a Universidade Federal Rural do Rio de Janeiro (UFRRJ) que, mais especificamente, em seu Instituto Três Rios no estado do Rio de Janeiro, criou, em 2010, o curso de bacharelado em Gestão Ambiental. Segundo o Projeto Pedagógico desse Curso (2013), as disciplinas multivariadas foram divididas entre os seguintes eixos: Engenharia, Exatas e da Terra; Biológicas; Jurídicas e Sociais; Multidisciplinar; Estudos Organizacionais e Optativas.

Diversas instituições de ensino implementaram os cursos superiores de Gestão Ambiental, em sua maioria, na modalidade de curso superior em 
tecnologia, porém é necessário lembrar que os cursos de Gestão Ambiental são recentes e estão, ainda, em processos de formação e estabilização de seus conceitos, metodologias e disciplinas.

Com base no amadurecimento ainda em processo dos cursos de Gestão Ambiental, é importante dizer que Leandro (2013, p. 80) destaca:

O primeiro curso brasileiro de tecnologia em gestão ambiental foi oferecido pelo Centro Federal de Educação Tecnológica Celso Suckow da Fonseca (CEFET-RJ) no ano de 1998 com duração de 3 anos. O primeiro curso oferecido na modalidade bacharelado, por sua vez, ocorreu no ano de 2002, na Universidade de São Paulo Escola Superior de Agricultura Luiz de Queiroz (USP-ESALQ).

\subsection{O contexto dos programas de acompanhamento de egressos}

A recomendação pelo acompanhamento de egressos foi dada na criação do Sistema de Consulta de Graduados (SCG), por meio da Portaria Normativa n. 91, de 31 de janeiro de 2014. O SCG é uma espécie de cadastro de todos os discentes já formados das instituições de ensino que possibilita a qualquer interessado conferir nesse Sistema se os dados cedidos pelos graduados estão em conformidade com os dados fornecidos pela instituição ao Censo Universitário.

O Sistema Nacional de Educação Superior (SINAES) avalia os três eixos do Ensino Superior - a avaliação das instituições, dos cursos e do desempenho dos estudantes. Alguns de seus instrumentos para tal procedimento são a autoavaliação, avaliação externa, avaliação dos cursos de graduação e instrumentos de informação, nos quais se incluem o censo e o cadastro. Portanto o acompanhamento de egressos é um processo contribuinte do instrumento de avaliação externa e auxilia na avaliação institucional do SINAES.

Além disso, a política de acompanhamento de egressos faz parte da estrutura da matriz das dimensões da avaliação externa de instituições de educação superior, encaixando-se na dimensão das políticas de atendimento aos estudantes. Dentro das diretrizes de avaliação externa das IES, o MEC (BRASIL, 2006, p.121) estabelece cinco critérios em relação à política de acompanhamento de egressos, apresentados a seguir: 


\section{Pontuações dos critérios considerados na avaliação da política de acompa- nhamento de egressos (BRASIL, 2006, p. 121)}

5- Quando existe política de acompanhamento do egresso de forma plenamente satisfatória; práticas consolidadas e institucionalizadas; há indicativos claros de organização e gestão com visão de futuro, ação direcionada; consistência nas práticas; política institucional assumida pelos atores internos e visível para a comunidade externa; quando existe na IES uma linha permanente de estudos e análises sobre alunos egressos, objetivando avaliar a qualidade do ensino e adequação dos currículos que contemple:

- mecanismos para a criação de uma base de dados, com informações atualizadas dos egressos; • mecanismos para a promoção de um relacionamento contínuo entre a instituição e seus egressos; • mecanismos para avaliar a adequação da formação do profissional para o mercado de trabalho; • mecanismos de utilização das opiniões dos egressos para aperfeiçoamento do processo de formação.

4- Quando existe política de acompanhamento do egresso de forma adequada; resulta ou expressa uma diretriz clara e definida para a ação dos atores acadêmicos, é de conhecimento da comunidade interna; aponta coerência, pertinência e congruência entre objetivos da IES; denota práticas institucionalizadas e difundidas.

3- Quando existe política de acompanhamento do egresso de forma razoável; resulta ou expressa, ainda que de forma incipiente, uma diretriz de ação; acessível à comunidade interna; denota programas e ações adequadas aos objetivos propostos pela IES; as práticas encontram-se em via de institucionalização.

2- Quando existe política de acompanhamento do egresso de forma eventual ou acidental.

1- Quando não existe política de acompanhamento do egresso.

Essas fundamentações baseadas em documentos produzidos pelo Ministério da Educação corroboram para a importância da criação e implementação de uma política bem estruturada e eficiente relacionada ao acompanhamento dos egressos.

Michelan et al. (2009) afirma que, apesar de as atividades de pesquisa e extensão também serem objetivos de uma Universidade, as instituições reconhecem como sua principal função: o ensino. E, por conta disso, as 
instituições de ensino devem exercer um controle das ações educacionais para com os discentes de forma a verificar a qualidade dessas ações.

Por isso a perspectiva dos egressos traz consigo contribuições de grande relevância para a sua instituição de ensino de origem - melhoria da qualidade e atualização dos cursos de graduação, extensão e pós-graduação -, em especial, as demandas da sociedade, a partir de suas realidades profissionais, acadêmicas e pessoais.

Em relação à região sudeste, em 2016, os cursos de Gestão Ambiental encontravam-se presentes em 161 instituições de ensino, sendo que três destes eram sequenciais, dois bacharelados e todo o restante, que contabiliza 156 instituições, era referente à modalidade de curso superior em tecnologia (quadro 1).

Quadro 1-Tipos de cursos de graduação em Gestão Ambiental (sequenciais, bacharelados e tecnológicos) existentes na região Sudeste

\begin{tabular}{|l|c|}
\hline Tipo de curso & Quantidade \\
\hline Sequenciais & 3 \\
\hline Bacharelados & 2 \\
\hline Tecnológicos & 156 \\
\hline
\end{tabular}

Fonte: Elaborado pelos autores.

Os cursos sequenciais encontravam-se especificamente no Centro Universitário Assunção (UNIFAI) na capital São Paulo, no Centro Universitário de Lins (UNILINS) no município de Lins, também no estado de São Paulo e em Minas Gerais, na Faculdade Pitágoras de Uberlândia (PIT), no município de Uberlândia. Destaca-se, inicialmente, das onze instituições de ensino públicas encontradas nesta pesquisa, apenas duas ofereciam o curso de Gestão Ambiental de forma presencial e na modalidade de bacharelado, enquanto as outras instituições privadas variavam entre cursos presenciais ou à distância e, predominantemente, cursos superiores em tecnologia. Em relação aos cursos de bacharelado, estes eram oferecidos, respectivamente na Universidade Federal Rural do Rio de Janeiro, em Três Rios, RJ, e na Universidade de São Paulo, nos municípios de Piracicaba e na capital São Paulo. 
Em 2015 eram, ao todo, 21 instituições de ensino privadas que ofereciam ensino à distância e 139 instituições variando entre públicas e privadas que ofereciam o curso na modalidade presencial como demonstrado no quadro 2.

Quadro 2 - Modalidades de cursos de graduação em Gestão Ambiental (ensino presencial e à distância) existentes na região Sudeste

\begin{tabular}{|l|c|}
\hline Modalidade & Quantidade \\
\hline Ensino Presencial & 21 \\
\hline Ensino à Distância & 139 \\
\hline
\end{tabular}

Fonte: Elaborado pelos autores.

Em termos percentuais, $13,12 \%$ dos cursos eram na modalidade de ensino à distância, e destes $100 \%$ constituíam-se de cursos superior em tecnologia. Dessa forma, $86,87 \%$ dos cursos eram presenciais divididos em: $2,14 \%$ sequenciais $1,43 \%$ bacharelados e $96,43 \%$ em cursos superiores em tecnologia.

\subsubsection{Rio de Janeiro}

No estado do Rio de Janeiro, 20 instituições ofereciam o curso de Gestão Ambiental na modalidade presencial distribuídas em 18 municípios, sendo cinco instituições públicas e o restante constituído de instituições privadas. Havia apenas uma instituição pública, a UFRRJ, que disponibilizava o único curso na modalidade bacharelado do estado, conforme apresentado no quadro 3.

Em relação ao ensino à distância, 11 instituições continham o curso superior em tecnologia de Gestão Ambiental em suas opções, distribuídos em 25 municípios do estado do Rio de Janeiro (quadro 3). 
Quadro 3-Tipos de cursos de graduação em Gestão Ambiental (sequenciais, bacharelados e tecnológicos) existentes no estado do Rio de Janeiro

\begin{tabular}{|l|c|}
\hline Tipo de curso & Quantidade \\
\hline Sequenciais & 0 \\
\hline Bacharelados & 1 \\
\hline Tecnológicos & 30 \\
\hline
\end{tabular}

Fonte: Elaborado pelos autores.

Quadro 4 - Modalidades de cursos de graduação em Gestão Ambiental (ensino presencial e à distância) existentes no estado do Rio de Janeiro

\begin{tabular}{|l|c|}
\hline Modalidade & Quantidade \\
\hline Ensino Presencial & 21 \\
\hline Ensino à Distância & 139 \\
\hline
\end{tabular}

Fonte: Elaborado pelos autores.

Em relação ao acompanhamento de egressos no Rio de Janeiro, foram encontrados Programas de Acompanhamento de Egressos gerais, isto é, envolvendo todos os cursos da instituição, em seis instituições da modalidade presencial, enquanto nas 14 instituições restantes não foram encontrados PAEs, conforme demonstrado no quadro 5.

A modalidade de ensino à distância, apresentou Programas de Acompanhamento de Egressos em cinco instituições e, nas outras sete instituições, não foi encontrado nenhum Programa, contudo, em uma dessas instituições, foram constatadas notícias sobre os ex-alunos, mas nenhum programa oficialmente elaborado (quadro 5). 
Quadro 5 - Programas de Acompanhamento de Egressos em relação às modalidades de ensino no estado do Rio de Janeiro

\begin{tabular}{|l|c|c|}
\hline PAE GERAL ENCONTRADO & SIM & NÃO \\
\hline Ensino Presencial & 6 & 14 \\
\hline Ensino à Distância & 5 & 7 \\
\hline
\end{tabular}

Fonte: Elaborado pelos autores.

\subsubsection{Minas Gerais}

Em Minas Gerais, 42 instituições de ensino ofereciam o curso de Gestão Ambiental na modalidade presencial distribuídas em 34 municípios do estado, sendo apenas um curso sequencial e o restante constituindo-se de cursos superiores em tecnologia, conforme exposto no quadro 6. Das 42 instituições, cinco eram públicas e as demais privadas.

Quadro 6 - Tipos de cursos de graduação em Gestão Ambiental (sequenciais, bacharelados e tecnológicos) existentes no estado de Minas Gerais

\begin{tabular}{|l|c|}
\hline Tipo de curso & Quantidade \\
\hline Sequenciais & 1 \\
\hline Bacharelados & 0 \\
\hline Tecnológicos & 57 \\
\hline
\end{tabular}

Fonte: Elaborado pelos autores.

Considerando-se a categoria de ensino à distância, havia 16 instituições privadas que ofereciam o curso superior em tecnologia de Gestão Ambiental distribuídas em 112 municípios do estado de Minas Gerais (quadro 7). 
Quadro 7 - Modalidades de cursos de graduação em Gestão Ambiental (ensino presencial e à distância) existentes no estado de Minas Gerais

\begin{tabular}{|l|c|}
\hline Modalidade & Quantidade \\
\hline Ensino Presencial & 42 \\
\hline Ensino à Distância & 16 \\
\hline
\end{tabular}

Fonte: Elaborado pelos autores.

Em relação ao levantamento realizado sobre a existência de Programas de Acompanhamento de Egressos, na modalidade presencial, foram encontrados 18 programas e nas 24 instituições restantes, nenhum Programa foi localizado.

Na modalidade de ensino à distância, nove instituições tiveram seus programas encontrados e, em sete instituições, não houve localização do programa. Contudo é importante destacar que, em uma destas instituições em que não havia a exposição do PAE, foram encontradas notícias relacionadas aos egressos (quadro 8):

Quadro 8 - Programas de Acompanhamento de Egressos em relação às modalidades de ensino no estado de Minas Gerais

\begin{tabular}{|l|c|c|}
\hline PAE GERAL ENCONTRADO & SIM & NÃO \\
\hline Ensino Presencial & 18 & 24 \\
\hline Ensino à Distância & 9 & 7 \\
\hline
\end{tabular}

Fonte: Elaborado pelos autores.

\subsubsection{Espírito Santo}

No estado do Espírito Santo, apenas quatro instituições privadas ofereciam o curso superior em tecnologia, distribuídas em 4 municípios diferentes (quadro 9). 
Quadro 9 -Tipos de cursos de graduação em Gestão Ambiental (sequenciais, bacharelados e tecnológicos) existentes no estado do Espírito Santo

\begin{tabular}{|l|c|}
\hline Tipo de curso & Quantidade \\
\hline Sequenciais & 0 \\
\hline Bacharelados & 0 \\
\hline Tecnológicos & 16 \\
\hline
\end{tabular}

Fonte: Elaborado pelos autores.

Em relação ao ensino à distância, o Estado possuía 12 instituições privadas, oferecendo todas estas o curso superior em tecnologia de Gestão Ambiental (quadro 10). Essas instituições possuíam polos distribuídos em 13 municípios.

Quadro 10 - Modalidades de cursos de graduação em Gestão Ambiental (ensino presencial e à distância) existentes no estado do Espírito Santo

\begin{tabular}{|l|c|}
\hline Modalidade & Quantidade \\
\hline Ensino Presencial & 4 \\
\hline Ensino à Distância & 12 \\
\hline
\end{tabular}

Fonte: Elaborado pelos autores.

No Espírito Santo, na modalidade presencial, foram encontrados Programas de Acompanhamento de Egressos no geral, em apenas uma instituição de ensino e, em três instituições, o programa não foi encontrado. Já na modalidade de educação à distância, seis instituições tiveram o PAE encontrado e, em outras seis, o PAE não foi localizado (quadro 11). 
Quadro 11 - Programas de Acompanhamento de Egressos em relação às modalidades de ensino no estado do Espírito Santo

\begin{tabular}{|l|c|c|}
\hline PAE GERAL ENCONTRADO & SIM & NÃO \\
\hline Ensino Presencial & 1 & 3 \\
\hline Ensino à Distância & 6 & 6 \\
\hline
\end{tabular}

Fonte: Elaborado pelos autores.

\subsubsection{São Paulo}

O estado de São Paulo possuía 73 instituições na modalidade presencial distribuídos em 43 municípios. Apenas uma instituição era pública e oferecia o único curso na categoria de bacharelado em Gestão Ambiental do estado de São Paulo. As demais instituições ofereciam cursos superiores em tecnologia de Gestão Ambiental. Ressalta-se que duas instituições possuíam o curso de Gestão Ambiental na modalidade sequencial, conforme apresentado no quadro 12.

Quadro 12-Tipos de cursos de graduação em Gestão Ambiental (sequenciais, bacharelados e tecnológicos) existentes no estado de São Paulo

\begin{tabular}{|l|c|}
\hline Tipo de curso & Quantidade \\
\hline Sequenciais & 2 \\
\hline Bacharelados & 1 \\
\hline Tecnológicos & 86 \\
\hline
\end{tabular}

Fonte: Elaborado pelos autores.

Em termos de ensino à distância, o estado de São Paulo superava todos os outros estados da região sudeste em relação à distribuição dos polos: eram, no total, 16 instituições privadas com cursos superiores em tecnologia de Gestão Ambiental distribuídas por 245 municípios do estado de São Paulo (quadro 13). 
Quadro 13 - Modalidades de cursos de graduação em Gestão Ambiental (ensino presencial e à distância) existentes no estado de São Paulo

\begin{tabular}{|l|c|}
\hline Modalidade & Quantidade \\
\hline Ensino Presencial & 73 \\
\hline Ensino à Distância & 16 \\
\hline
\end{tabular}

Fonte: Elaborado pelos autores.

Nesse estado, o Programa de Acompanhamento de Egressos foi encontrado em 31 instituições da modalidade presencial, duas instituições não tiveram seus sites encontrados e uma apresentou problemas de carregamento em sua página. Na modalidade de educação à distância, o programa foi encontrado em dez instituições e nas seis restantes, nenhum PAE foi localizado.

Quadro 14 - Programas de Acompanhamento de Egressos em relação às modalidades de ensino no estado de São Paulo

\begin{tabular}{|l|c|c|}
\hline PAE GERAL ENCONTRADO & SIM & NÃO \\
\hline Ensino Presencial & 31 & 41 \\
\hline Ensino à Distância & 10 & 6 \\
\hline
\end{tabular}

Fonte: Elaborado pelos autores.

Os estados de São Paulo, Minas Gerais e Rio de Janeiro apresentaram-se, respectivamente, como os que possuem a maioria de cursos superiores em Gestão Ambiental, independentemente de suas modalidades. Isso provavelmente seja decorrente da necessidade de atendimento aos polos econômicos que representam, no caso de São Paulo e Rio de Janeiro, e à sua dimensão territorial, no caso do estado de Minas Gerais.

A pesquisa efetivada corrobora com o que Leandro (2013 p. 99) observou em seu estudo: São Paulo concentra a maior quantidade de cursos superiores de tecnologia em gestão ambiental da região sudeste (56\% dos cursos da região sudeste). O pesquisador atribuiu esse dado ao alto nível de industrialização e à parcela de participação do estado no Produto Interno 
Bruto nacional, que contribuía com 30\% da produção total de bens e serviços no país.

É perceptível que a modalidade de cursos superiores em tecnologia de Gestão Ambiental em 2016 foi dominante na região sudeste. O motivo desse fato constitui-se em um incentivo para a realização de novas pesquisas. Isso com o intuito de constatar diretamente as instituições de ensino, que implementaram essa modalidade e questionar sobre as influências para a efetivação dessa escolha.

Além disso, talvez essa realidade se deva ao fato de que, segundo Leandro (2013), os cursos de bacharelado em Gestão Ambiental ainda não possuem Diretrizes Curriculares Nacionais (DCN), o que causa uma problemática em termos de definição de objetivos e características enquanto os cursos superiores em tecnologia tiveram suas Diretrizes a provadas em 2002.

Todas as instituições de ensino que possuem acompanhamento de egressos, não possuem o programa especificamente para o curso superior em Gestão Ambiental. Todos os programas encontrados foram elaborados de forma generalizada, ao menos nas apresentações que são retratadas aos visitantes das páginas eletrônicas. Supõe-se que haja divisão por cursos dentro da página de egressos, que, por sua vez, é apresentada somente aos cadastrados.

Na região sudeste, não foram encontrados Programas de Acompanhamento de Egressos específicos aos cursos de Gestão Ambiental, todos os programas encontrados continham dados generalizados, ou seja, de todos os cursos sem diferenciação. Porém é importante considerar que os cursos superiores em Gestão Ambiental são recentes e ainda se encontram em fase de consolidação.

\section{CONCLUSÃO}

A partir de todas as informações apresentadas acima, nota-se que a ferramenta de acompanhamento de egressos é extremamente importante como mecanismo de controle de qualidade dos cursos superiores e possibilidades de melhorias. Entretanto é ainda pouco conhecida pelos coordenadores que gerenciam os cursos superiores. 
Nesse contexto, faz-se necessário maior divulgação desse instrumento e de sua importância visto que auxilia na melhoria contínua do ensino superior. As instituições de ensino e os órgãos públicos educacionais como o Ministério da Educação devem fornecer orientações claras e objetivas referentes ao programa, de forma a esclarecer sua implementação aos coordenadores de cada curso oferecido.

É importante esclarecer a importância e a necessidade do acompanhamento de egressos também para os discentes dos cursos superiores, incentivando-os, quando egressos, a participarem desse programa, respondendo aos questionários e participando das discussões sobre o curso. Algumas instituições oferecem benefícios para manter o egresso próximo à academia, como a liberação do uso da biblioteca e descontos em eventos, por exemplo.

A relação universidade-egresso não deve ser finalizada após a formação do profissional, e é fundamental que ambos compreendam a suma importância desse relacionamento para a melhoria do curso, considerando sempre a contribuição dessa melhoria para o mercado de trabalho.

\section{REFERÊNCIAS}

BARBIERI, José Carlos. Gestão ambiental empresarial: conceitos, modelos e instrumentos. 1. ed. São Paulo: Saraiva, 2007.

BRASIL. Ministério da Educação. Portaria Normativa n. 91, 31 de julho de 2014. Institui o Sistema de Consulta de Graduados-SCG. Diário Oficial [da] República Federativa do Brasil, Poder Executivo, Brasília, DF, 4 fev. 2014. Seção 1, p. 5.

BRASIL. Ministério da Educação. Portaria Normativa n. 92, 31 de janeiro de 2014. Aprova, em extrato, os indicadores do Instrumento de Avaliação Institucional Externa para os atos de credenciamento, recredenciamento e transformação de organização acadêmica, modalidade presencial, do Sistema Nacional de Avaliação da Educação Superior- SINAES. Diário Oficial [da] República Federativa do Brasil, Poder Executivo, Brasília, DF, 4 fev. 2014. Seção 1, p. 5.

BRASIL. Ministério da Educação. Avaliação Externa das Instituições de Educação Superior. Diretrizes e Instrumentos. Brasília: MEC, 2006. 180p. 
BRASIL. Conselho Nacional de Meio Ambiente. Resolução n. 306, 5 de julho de 2002. Estabelece os requisitos mínimos e o termo de referência para realização de auditorias ambientais. Diário Oficial [da] República Federativa do Brasil, Poder Executivo, Brasília, DF, 19 jul. 2002. p. 75-6.

CADASTRO E-MEC DE INSTITUIÇÕES E CURSOS DE EDUCAÇÃO SUPERIOR. Disponível em: http://emec.mec.gov.br/. Acesso em: 10 mar. 2016.

LEANDRO, Luiz Alberto de Lima. A formação superior de Gestores Ambientais no Brasil: contribuição para a formulação de Diretrizes Curriculares Nacionais. 2013. 282p. Tese (Doutorado em Meio Ambiente) - Universidade do Estado do Rio de Janeiro (UERJ), Rio de Janeiro, 2013.

LOUSADA, Ana Cristina Zenha; MARTINS Gilberto de Andrade. Egressos como fonte de informação à gestão dos cursos de Ciências Contábeis. Revista Contabilidade e Finanças, São Paulo, v. 16, n. 37, p. 73-84, jan./abr. 2005.

MICHELAN, Luciano Sérgio et al. Gestão de egressos em instituições de ensino superior: possibilidades e potencialidades. In: COLÓQUIO INTERNACIONAL SOBRE GESTÃO UNIVERSITÁRIA NA AMÉRICA DO SUL, 9., nov. 2009, Florianópolis, SC. p. 1-16. Disponível em: https://repositorio.ufsc.br/bitstream/handle/123456789/36720/ Gest\%C3\%A3o\%20de\%20egressos\%20em\%20institui\%C3\%A7\%C3\%B5es\%20 de\%20ensino\%20superior\%20 Possibilidades\%20e\%20pontencialidades. pdf?sequence $=1$

MILLER JUNIOR, G. Tyler. Ciência ambiental. 11. ed. São Paulo: Cengage Learning, 2014.

SÁNCHEZ, Luis Enrique. Avaliação de impacto ambiental: conceitos e métodos. 2. ed. São Paulo: Oficina de Textos, 2013.

SISTEMA DE CONSULTA DE GRADUADOS. Disponível em: http://consultadegraduados. mec.gov.br/principal/comofunciona. Acesso em: 5 mar. 2016.

UFRRJ. Projeto Pedagógico do Curso de Gestão Ambiental. Deliberação n. 40, 26 de abril de 2013. 
